\title{
Extra Dimensions and Neutrinoless Double Beta Decay Experiments
}

\author{
Marek Góźdź, 1, W Wiesław A. Kamiński, 1, 田 and Amand Faessler 2, 团 \\ ${ }^{1}$ Theoretical Physics Department, Maria Curie-Sktodowska University, Lublin, Poland \\ ${ }^{2}$ Institute für Theoretische Physik, Universität Tübingen, \\ Auf der Morgenstelle 14, D-72076 Tübingen, Germany
}

\begin{abstract}
The neutrinoless double beta decay is one of the few phenomena, belonging to the non-standard physics, which is extensively being sought for in experiments. In the present paper the link between the half-life of the neutrinoless double beta decay and theories with large extra dimensions is explored. The use of the sensitivities of currently planned $0 \nu 2 \beta$ experiments: DAMA, CANDLES, COBRA, DCBA, CAMEO, GENIUS, GEM, MAJORANA, MOON, CUORE, EXO, and XMASS, gives the possibility for a non-direct 'experimental' verification of various extra dimensional scenarios. We discuss also the results of the Heidelberg-Moscow Collaboration. The calculations are based on the Majorana neutrino mass generation mechanism in the Arkani-Hamed-DimopoulosDvali model.
\end{abstract}

PACS numbers: $11.10 . \mathrm{Kk}, 12.60 .-\mathrm{i}, 14.60 . \mathrm{St}, 14.60 . \mathrm{Pq}$

Keywords: neutrino mass, extra dimensions, ADD model, neutrinoless double beta decay

\section{INTRODUCTION}

The standard model (SM), despite being very successful, has many drawbacks and cannot be regarded as the ultimate theory of elementary particles and their interactions. One of the most severe are the big number of free parameters and the hierarchy problem. The idea of going beyond the SM is old, but only recently has been fully backed up by experimental evidence of neutrino oscillations [1 5]. This observation clearly calls for a more fundamental theory, which will extend the SM. One of the best candidates, which cures most of the SM's weaknesses, is the theory of superstrings. Within this theory one gets rid of the problematic point-like nature of particles, therefore removing ultraviolet divergences. What is more, there is no need to introduce and fit to experiments so many free parameters (more than 20 in the SM). The string theory is a consistent, divergent-free quantum field theory. It allows also to generate, in a natural way, a spin2 massless field, which represents the graviton, therefore unifying gravity and electroweak interactions.

However, the string theory requires two new features for consistent formulation: the supersymmetry and additional spatial dimensions. Supersymmetry introduces new kind of operators, which change the spin of particles by $1 / 2$. Therefore a boson may be turned into a fermion and vice versa, which results in unification of forces and matter. The idea of additional spatial dimensions sound even more strange. Because these two phenomena are not observed in the low energy domain, the supersymmetric particles must be heavier than our current experimental capabilities, and extra dimensions much smaller than our ability to observe them. They may form closed, curled

\footnotetext{
*Electronic address: mgozdz@kft.umcs.lublin.pl

${ }^{\dagger}$ Electronic address: kaminski@neuron.umcs.lublin.pl

‡Electronic address: amand.faessler@uni-tuebingen.de
}

shapes with tiny radii (the so-called Calabi-Yau shapes) which has very small influence on our life. It is, however, possible that the extra dimensions are open, large, and the difficulty in observing them has a different basis.

There are three main models used in building theories with large extra dimensions. Firstly, we have the description based on the original Kaluza-Klein approach [6, 7], which predicts a load of new particles, the so-called towers of KK excitations. Secondly, there are models formulated by Randall and Sundrum (RS models) 8, 9], in which the extra dimensions are compactified on orbifolds with a $Z_{2}$ symmetry. Last but not least, there are propositions of Arkani-Hammed, Dimopoulos and Dvali (ADD models) 10 14], in which the standard model particles are trapped on a 3D brane, which in turn floats in a higher dimensional bulk. In what follows we will use the ADD approach.

As mentioned above, the main evidence for physics beyond the SM are the oscillations of neutrinos. According to most theories, neutrino oscillations imply a non-zero neutrino mass and a difference between the flavor and mass eigenstates of neutrinos. The physical mechanism generating this difference, as well as masses, remain still an open issue.

Assuming the existence of supersymmetry, or nonstandard physics in general, one may expect the phenomena, normally forbidden by the symmetries of SM. One of these is the neutrinoless channel of double beta decay $(0 \nu 2 \beta)$ [15]. This decay, still not confirmed, is the only experimental possibility of determining the nature of neutrino (Majorana or Dirac particle). It may also help in obtaining the absolute mass of these particles, since the oscillation experiments are sensitive to differences of masses squared only.

In our earlier work [16, 17] we have shown, that it is possible to relate the parameters describing extra dimensions to the half-life of the $0 \nu 2 \beta$ decay. In the present paper we continue this topic with the analysis of the data provided by the Heidelberg-Moscow Collaboration (H- 
M) as well as sensitivities of planned $0 \nu 2 \beta$ experiments, from the point of view of large extra dimensions.

In the next section we recall the necessary information about the ADD model of large extra dimensions. In the following section we shortly discuss the formula describing the half-life of neutrinoless double beta decay. After that, we arrive at the final formula and present and discuss the results. A summary follows at the end.

\section{EXTRA DIMENSIONS}

In the ADD approach the space can be divided into two main parts. One is the so-called bulk, a $3+$ $n$-dimensional space in which the gravity propagates. Besides gravitons, it may be also populated with other particles and fields, which are not contained in the standard model. Floating in the bulk, there is at least one 3 -dimensional brane, an object which is predicted by the string theory as a higher-dimensional generalization of the string. The standard model is assumed to be confined on such brane. It means that all the SM interactions are propagating only within the brane. The same goes to fermions, which must also be restricted to live on the brane. The just presented setting immediately explains the weakness of gravity in our universe, thus solving the hierarchy problem. Namely, using for example the generalized Gauss Law, one arrives at the so-called reduction formula [13]

$$
M_{P l}^{2} \sim R^{n} M_{*}^{2+n},
$$

where $M_{P l} \sim 10^{28} \mathrm{eV}$ is the Planck mass, $M_{*}$ is the true scale of gravity, and $R$ is the assumed common compactification radius of extra dimensions. One sees that, by properly adjusting the values of $R$ and $n$, it is possible to lower the true scale of gravity to the electroweak scale $\sim 1 \mathrm{TeV}$, thus getting rid of the hierarchy problem. This is the main motivation for the ADD approach.

Introducing a second brane, parallel to ours, one may generate, under special conditions, a Majorana neutrino mass term. Let us denote the coordinates by $\left\{x^{\mu}, y^{m}\right\}$, where $\mu=0 \ldots 3$ labels the ordinary space-time coordinates and $m=1 \ldots n$ labels the extra dimensions. By identifying $y \sim y+2 \pi R$ we compactify the extra dimensions on circles. (From now on we will drop the indices $\mu$ and $m$ for simplicity.)

Let us assume [13, 14] that lepton number is conserved on our brane, located at $y=0$, but maximally broken on the other one, placed on $y=y_{*}$. The breaking occurs in a reaction where a particle $\chi$, with lepton number $L=2$ and mass $m_{\chi}$, escapes the other brane into the bulk. This particle, called the messenger, may interact with our brane and transmit to us the information about lepton number breaking.

To be more specific, let us introduce a field $\phi_{L=2}$ located on the other brane, whose vacuum expectation value (vev) breaks the lepton number. What is more, it acts as a source for the bulk messenger field $\chi$ and "shines" it everywhere, in particular also on our brane. The strength of the shined $\chi$ is in a natural way suppressed by the distance $r$ between branes, and therefore one can write for the messenger

$$
\langle\chi\rangle=\langle\phi\rangle \Delta_{n}(r),
$$

where $\Delta_{n}(R)$ is the $n$-dimensional propagator given by

$$
\begin{aligned}
\Delta_{2}(R) & \sim \begin{cases}-\log \left(R m_{\chi}\right), & \left(R m_{\chi} \ll 1\right) \\
\frac{e^{-R m_{\chi}}}{\sqrt{R m_{\chi}}}, & \left(R m_{\chi} \gg 1\right)\end{cases} \\
\Delta_{n>2}(R) & \sim \begin{cases}\frac{1}{R^{n-2}}, & \left(R m_{\chi} \ll 1\right) \\
\frac{e^{-R m_{\chi}}}{R^{n-2}}, & \left(R m_{\chi} \gg 1\right)\end{cases}
\end{aligned}
$$

We introduce a lepton field $l(x)$ and a Higgs scalar field $h(x)$ localized on our brane. They can interact with the messenger and the interaction is given by the following Lagrangian [14]:

$$
\begin{aligned}
M_{*}^{n-1} \mathcal{L}^{i n t} \sim & \int d^{4} x^{\prime}\langle\phi\rangle \chi\left(x^{\prime}, y_{*}\right)+ \\
& \int d^{4} x \alpha\left[l(x) h^{*}(x)\right]^{2} \chi(x, 0),
\end{aligned}
$$

where the first part represents the lepton number violation, occurring on the other brane, and the second part is responsible for the interaction between SM fields and the messenger on our brane. Let us assume for simplicity, that the second brane is as far away from ours as possible, i.e. the distance between the branes is approximately equal to $R$, the compactification radius. After spontaneous symmetry breaking we substitute (2) into (5), write the Higgs field in terms of its vev $v$, and identify $l$ with $\nu_{L}$. We arrive at a mass term of the Majorana form $m_{M a j} \nu_{L}^{T} \nu_{L}$ with the mass given approximately by 14]:

$$
m_{M a j} \sim \frac{v^{2} \Delta(r)}{M_{*}^{n-1}} .
$$

which, using Eq. (1), may be rewritten as [16]:

$$
m_{M a j} \sim v^{2} R^{\frac{n(n-1)}{n+2}} M_{P l}^{\frac{2(1-n)}{n+2}} \Delta_{n}(R),
$$

with $v^{2}=(174 \mathrm{GeV})^{2} \sim 10^{22} \mathrm{eV}^{2}$ being the Higgs boson vev.

\section{NEUTRINOLESS DOUBLE BETA DECAY}

Neutrinoless double beta decay $(0 \nu 2 \beta)$ is a process in which a nucleus undergoes two simultaneous beta decays without emission of neutrinos

$$
A(Z, N) \rightarrow A(Z+2, N-2)+2 e^{-} .
$$

It requires neutrino to be a Majorana particle, so that two neutrinos emitted in beta decays annihilate with each 
other. It is readily seen that this process violates the lepton number by two units, thus it is forbidden in the framework of SM. The $0 \nu 2 \beta$ decay has been claimed to be observed [18], but this information has not yet been confirmed and has met some strong criticism from the community (see e.g. [19, 20]). In any case, even the limit for non-observability of this decay sets valuable constraints on the shape of physics beyond the SM. It is the main tool to verify the nature of neutrino (Dirac or Majorana particle). It may also help in determining the absolute value of the neutrino mass.

Ignoring the contributions from right-handed weak currents, the half-life of $0 \nu 2 \beta$ can be written in the form [15]:

$$
T_{1 / 2}=\mathcal{M}_{\text {spec }}^{-1} \frac{m_{e}^{2}}{\left\langle m_{\nu}\right\rangle^{2}} .
$$

One can write the same for any performed or planned $0 \nu 2 \beta$ experiment, replacing the relevant values with the sensitivities of the experiments

$$
T_{1 / 2}^{\text {expt. }}=\mathcal{M}_{\text {spec }}^{-1} \frac{m_{e}^{2}}{\left\langle m_{\nu}\right\rangle_{\text {expt. }}^{2}},
$$

In relations (9) and (10) $\mathcal{M}_{\text {spec }}$ is the nuclear matrix element for a specific nucleus which can be calculated within certain nuclear models, and $m_{e}$ is the electron mass. The so-called effective neutrino mass $\left\langle m_{\nu}\right\rangle$ is defined by the relation

$$
\begin{aligned}
\left\langle m_{\nu}\right\rangle & =\left|\sum_{i} m_{i} U_{e i}^{2}\right| \\
& \left.\Rightarrow\left|m_{1}\right| U_{e 1}\right|^{2} \pm m_{2}\left|U_{e 2}\right|^{2} \pm m_{3}\left|U_{e 3}\right|^{2} \mid,
\end{aligned}
$$

where $U$ is the neutrino mixing matrix and $m_{i}$ are neutrino mass eigenvalues. The last formulation assumes CP invariance. One sees from this equation that it is possible to identify $\left\langle m_{\nu}\right\rangle$ with the ee entry of neutrino mass matrix in the flavor basis

$$
\left\langle m_{\nu}\right\rangle=m_{e e},
$$

which is given exactly by the superposition of mass eigenvalues from Eq. (11).

In the next section the link between $T_{1 / 2}$ and the parameters describing extra dimensions will be established.

\section{RESULTS}

In the calculations we have neglected the contribution coming from the third neutrino in the mass basis, setting $\left|U_{e 3}\right|^{2} m_{3}=0$. This is justified by the results of CHOOZ experiment [26, 27], which showed that there is a negligible admixture of $\nu_{e}$ in the third mass eigenstate (less than $3 \%$; most of the analyses place the value of $\left|U_{e 3}\right|^{2}$ between 0 and 0.05 ). The remaining two mass eigenstates
TABLE I: Sensitivities of some $0 \nu 2 \beta$ experiments planned for the future. The half-life $T_{1 / 2}^{\text {expt. }}$ is expressed in years, while effective neutrino mass in $\mathrm{eV} ; \xi=T_{1 / 2}^{\text {expt. }}\left\langle m_{\nu}\right\rangle_{\text {expt. }}^{2}$. Values taken from Refs. 21 25].

\begin{tabular}{lccc}
\hline \hline & $\begin{array}{c}T_{1 / 2}^{\text {expt. }} \\
{[\mathrm{y}]}\end{array}$ & $\begin{array}{c}\left\langle m_{\nu}\right\rangle_{\text {expt. }} \\
{[\mathrm{eV}]}\end{array}$ & $\begin{array}{c}\xi \\
{\left[\mathrm{y} \mathrm{eV}^{2}\right]}\end{array}$ \\
expt. & $1.2 \times 10^{24}$ & 2.3 & $6.3 \times 10^{24}$ \\
\hline DAMA $\left({ }^{136} \mathrm{Xe}\right)$ & $3 \times 10^{27}$ & 0.044 & $5.8 \times 10^{24}$ \\
MAJORANA $\left({ }^{76} \mathrm{Ge}\right)$ & $4 \times 10^{28}$ & 0.012 & $5.7 \times 10^{24}$ \\
EXO 10t $\left({ }^{136} \mathrm{Xe}\right)$ & $7 \times 10^{27}$ & 0.028 & $5.5 \times 10^{24}$ \\
GEM $\left({ }^{76} \mathrm{Ge}\right)$ & $1 \times 10^{28}$ & 0.023 & $5.3 \times 10^{24}$ \\
GENIUS $\left({ }^{76} \mathrm{Ge}\right)$ & $1 \times 10^{26}$ & 0.2 & $4.0 \times 10^{24}$ \\
CANDLES $\left({ }^{48} \mathrm{Ca}\right)$ & $1 \times 10^{27}$ & 0.058 & $3.4 \times 10^{24}$ \\
MOON $\left({ }^{100} \mathrm{Mo}\right)$ & $3 \times 10^{26}$ & 0.10 & $3.0 \times 10^{24}$ \\
XMASS $\left({ }^{136} \mathrm{Xe}\right)$ & $2 \times 10^{26}$ & 0.10 & $2.0 \times 10^{24}$ \\
CUORE $\left({ }^{130} \mathrm{Te}\right)$ & $1 \times 10^{24}$ & 1 & $1.0 \times 10^{24}$ \\
COBRA $\left({ }^{116} \mathrm{Cd}\right)$ & $2 \times 10^{26}$ & 0.07 & $9.8 \times 10^{23}$ \\
DCBA $\left({ }^{100} \mathrm{Mo}\right)$ & $3 \times 10^{26}$ & 0.04 & $4.8 \times 10^{23}$ \\
DCBA $\left({ }^{82} \mathrm{Se}\right)$ & $1 \times 10^{27}$ & 0.02 & $4.0 \times 10^{23}$ \\
CAMEO $\left({ }^{116} \mathrm{Cd}\right)$ & $1 \times 10^{26}$ & 0.02 & $4.0 \times 10^{22}$ \\
DCBA $\left({ }^{150} \mathrm{Nd}\right)$ &
\end{tabular}

can be treated as nearly degenerate, with $m_{1} \approx m_{2}[28$ ]. Within these approximations one may express $m_{e e}$ as a function of $m_{1}$ and two mixing angles in the following form [28]:

$$
m_{e e}^{2}=\left[1-\sin ^{2}\left(2 \theta_{\text {Solar }}\right) \sin ^{2}\left(\phi_{12} / 2\right)\right] m_{1}^{2} \equiv \kappa^{-1} m_{1}^{2},
$$

where $\sin ^{2}\left(2 \theta_{\text {Solar }}\right) \approx 0.82$; this value takes into account the KamLand results [1-3, 29]. By CP symmetry conservation, the relative phase factor $\phi_{12}$ takes values either 0 or $\pi / 2$. For $\phi_{12}=0, m_{e e}=m_{1}$. The more interesting case involves the mixing angle $\theta_{\text {Solar }}$ therefore we chose the value $\phi_{12}=\pi / 2$ having

$$
m_{e e}^{2}=1.69 m_{1}^{2},
$$

In fact the exact value of $\kappa$ is irrelevant in our discussion and the whole coefficient can in principle be set to one. What we are interested in is the order of magnitude of the half-life of $0 \nu 2 \beta$ decay.

By rewriting $T_{1 / 2}$ in terms of $T_{1 / 2}^{e x p t .}$ we get rid of the unwanted nuclear matrix elements

$$
T_{1 / 2}=T_{1 / 2}^{\text {expt. }} \frac{\left\langle m_{\nu}\right\rangle_{\text {expt. }}^{2}}{\left\langle m_{\nu}\right\rangle^{2}}=\xi\left\langle m_{\nu}\right\rangle^{-2}
$$

where we have gathered all experimental values in the parameter $\xi=T_{1 / 2}^{\text {expt. }}\left\langle m_{\nu}\right\rangle_{\text {expt. }}^{2}$. Eqs. (12) and (14) allow to rewrite this expression as

$$
T_{1 / 2}=\xi m_{e e}^{-2}=\xi \kappa m_{1}^{-2} .
$$

Finally, we assume that neutrinos are Majorana particles in order to discuss the neutrinoless double beta decay. 
Since we are left with only one independent mass, we may replace $m_{1}$ with the expression for Majorana neutrino mass, Eq. (7), finishing with

$$
\begin{aligned}
T_{1 / 2} & =\kappa \xi v^{-4} R^{2 n(1-n) / n+2} \\
& \times M_{P l}^{4(n-1) / n+2}\left[\Delta_{n}(R)\right]^{-2} .
\end{aligned}
$$

One sees that Eq. (17) consists of two parts. The first one is connected with $0 \nu 2 \beta$ experiments, for which the relevant values are presented in Tab. (1). We have included tha values as given by various collaboration assuming, that these represent the best sensitivity of the projects. Therefore the effective neutrino mass, which depends on the nuclear matrix elements, may be different for the same isotope. However the calculations of the matrix elements using various approaches give on average a change in $\left\langle m_{\nu}\right\rangle 200-300 \%$ which is well below the level of accuracy of our discussion.

The second part of Eq. (17) contains parameters of the extra dimensions. At this point we need to include constraints coming from other sources, like supernova and neutron star data [30 34], and cosmological models [35, 36]. Altogether, one of the most complete limits have been derived in Ref. [32] and read:

$$
\begin{array}{cl}
R<1.5 \times 10^{-7} \mathrm{~mm} & \text { for } n=2, \\
R<2.6 \times 10^{-9} \mathrm{~mm} & \text { for } n=3, \\
R<3.4 \times 10^{-10} \mathrm{~mm} & \text { for } n=4 .
\end{array}
$$

Taking into account bounds Eqs. (18) $-(20)$ the formula for $T_{1 / 2}$ becomes an inequality. Explicitly Eq. (17) takes the following forms of lower bounds on the half-life of $0 \nu 2 \beta$ :

Case $n=2$

$$
\begin{aligned}
T_{1 / 2}>1.11 \times 10^{-15} \xi^{\prime}\left(\log \left(R m_{\chi}\right)\right)^{-2}, & \left(R m_{\chi} \ll 1\right) ; \\
T_{1 / 2}>1.11 \times 10^{-15} \xi^{\prime} e^{2 R m_{\chi}} R m_{\chi}, & \left(R m_{\chi} \gg 1\right) .
\end{aligned}
$$

Case $n=3$

$$
\begin{aligned}
T_{1 / 2}>0.039 \xi^{\prime}, & \left(R m_{\chi} \ll 1\right) ; \\
T_{1 / 2}>0.039 \xi^{\prime} e^{2 R m_{\chi}}, \quad & \left(R m_{\chi} \gg 1\right) .
\end{aligned}
$$

Case $n=4$

$$
\begin{aligned}
T_{1 / 2}>1.69 \times 10^{11} \xi^{\prime}, & & \left(R m_{\chi} \ll 1\right) ; \\
T_{1 / 2}>1.69 \times 10^{11} \xi^{\prime} e^{2 R m_{\chi}} . & & \left(R m_{\chi} \gg 1\right) .
\end{aligned}
$$

We have denoted $\xi^{\prime}=\xi /\left(1 \mathrm{eV}^{2}\right)$. In the above calculations the value $\kappa=1.69$ has been used, which is consistent with the KamLand results [29]. The inequalities represent lower bounds on the half-life. The true value of $T_{1 / 2}$ may be much bigger than the bounds itself. We would like to stress, that the following discussion is valid only under our assumptions, i.e. we live in a brane world and generate neutrino masses according to the ADD suggestion. If not, our bounds on $T_{1 / 2}$ are not valid. Inserting $\xi$ corresponding to various experiments one obtains specific values of $T_{1 / 2}$. If a positive signal will be recorded by that collaboration, it may be compared with our values to estimate the values of $n, R$ and $R m_{\chi}$.

Let us start with the discussion of the claim of evidence of $0 \nu 2 \beta$ decay. Klapdor et. al. in Ref. [18] reported a positive signal for the $0 \nu 2 \beta$ transition in ${ }^{76} \mathrm{Ge}$ and, after reanalyzing the data, announced the following values at more than $99.9 \%$ confidence level [37]:

$$
T_{1 / 2}^{H-M}=1.19 \times 10^{25} \mathrm{y}, \quad\left\langle m_{\nu}\right\rangle_{H-M}=0.44 \mathrm{eV},
$$

which corresponds to $\xi=2.3 \times 10^{24} \mathrm{y} \mathrm{eV}^{2}$. This claim, although not confirmed, has not been withdrawn, so it deserves a careful analysis. The results are presented in Fig. 1 .

One sees that for a light messenger particle, the results do not depend on $R m_{\chi}$, except for $n=2$, where the dependence is very weak. In fact they are even independent of $R$ and $m_{\chi}$ separately. Obtaining constant values for that case (for $n \geq 3$ ) is a general feature of the model, as will be seen in further discussion. Assuming that the $\mathrm{H}-$ $\mathrm{M}$ values correctly describe $0 \nu 2 \beta$ decay, and taking into account possible errors, the closest match will be $n=3$. The $n=2$ case is practically ruled out, since the sensitivities of the experiments exceeded the obtained value by more than 15 orders of magnitude, and all of them reported negative results. Another possible scenario is the $n=4$, but its verification will have to wait for more powerful experiments than those planned today.

In the case of heavy messenger, the first solution is $n=2$ and $R m_{\chi} \approx 17$. If the $\mathrm{H}-\mathrm{M}$ results were confirmed, this possibility is promising. Another choice could be $n=3$ and $R m_{\chi}$ being close to 1 . This, however, puts a question mark on our prediction, since we have used the asymptotic al forms of the propagator, which are not valid for $R m_{\chi} \approx 1$. More extra dimensions blow the halflife to extremely huge values, making the discussion at this stage meaningless.

The H-M data's $\xi$ value places itself in the middle of considered projects. Let us now say a few words about the currently planned $0 \nu 2 \beta$ experiments. The results for the DCBA experiment, which has the smallest $\xi$ value, and for DAMA experiments with the biggest $\xi$ are depicted on Figs. 2 and 3 respectively. For the remaining projects the results are summarized in Tabs. III and III. All the values presented in the tables are bounds from below on the $0 \nu 2 \beta$ half-life. In any case one should always bear in mind the upper limit on $R$ and from the value of $R m_{\chi}$ deduce the appropriate mass of the messenger particle.

The results for $n=2$ are summarized in Tab. II The case of light messenger is excluded by the negative results of $0 \nu 2 \beta$ experiments, which sensitivities have exceeded the given threshold by more than 15 orders of magnitude. In the case of heavy messenger, the quantity $R m_{\chi}$ turns out to be equal to at least 15 . This value matches well the $R m_{\chi} \approx 17$ obtained for $\mathrm{H}-\mathrm{M}$ experiment.

Case $n=3$ is presented in Tab. III for heavy messenger. One sees that this case is not limited by the ex- 

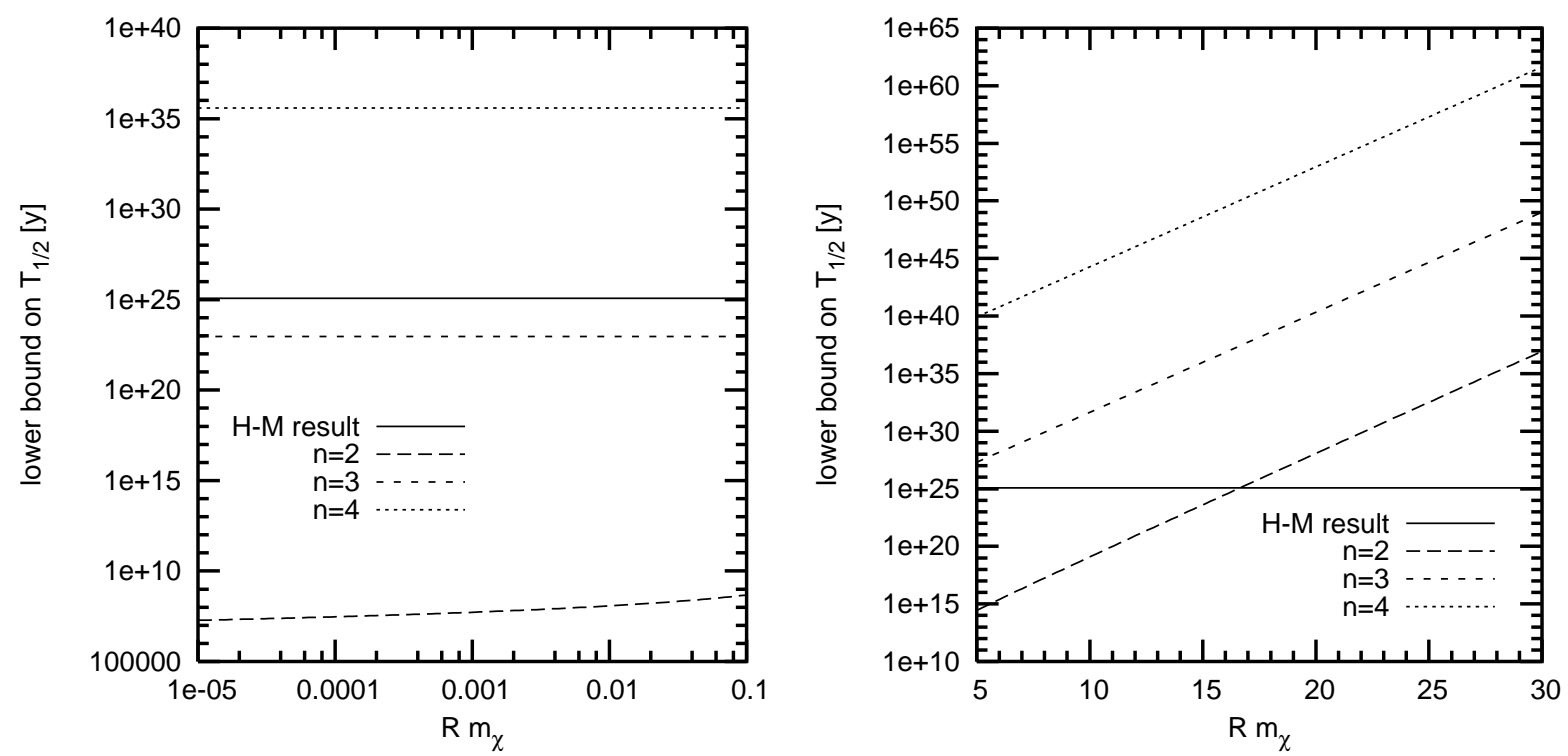

FIG. 1: Lower bounds on the half-life of neutrinoless double beta decay in the case of light (left panel) and heavy (right panel) messenger particle for the Heidelberg-Moscow experiment.
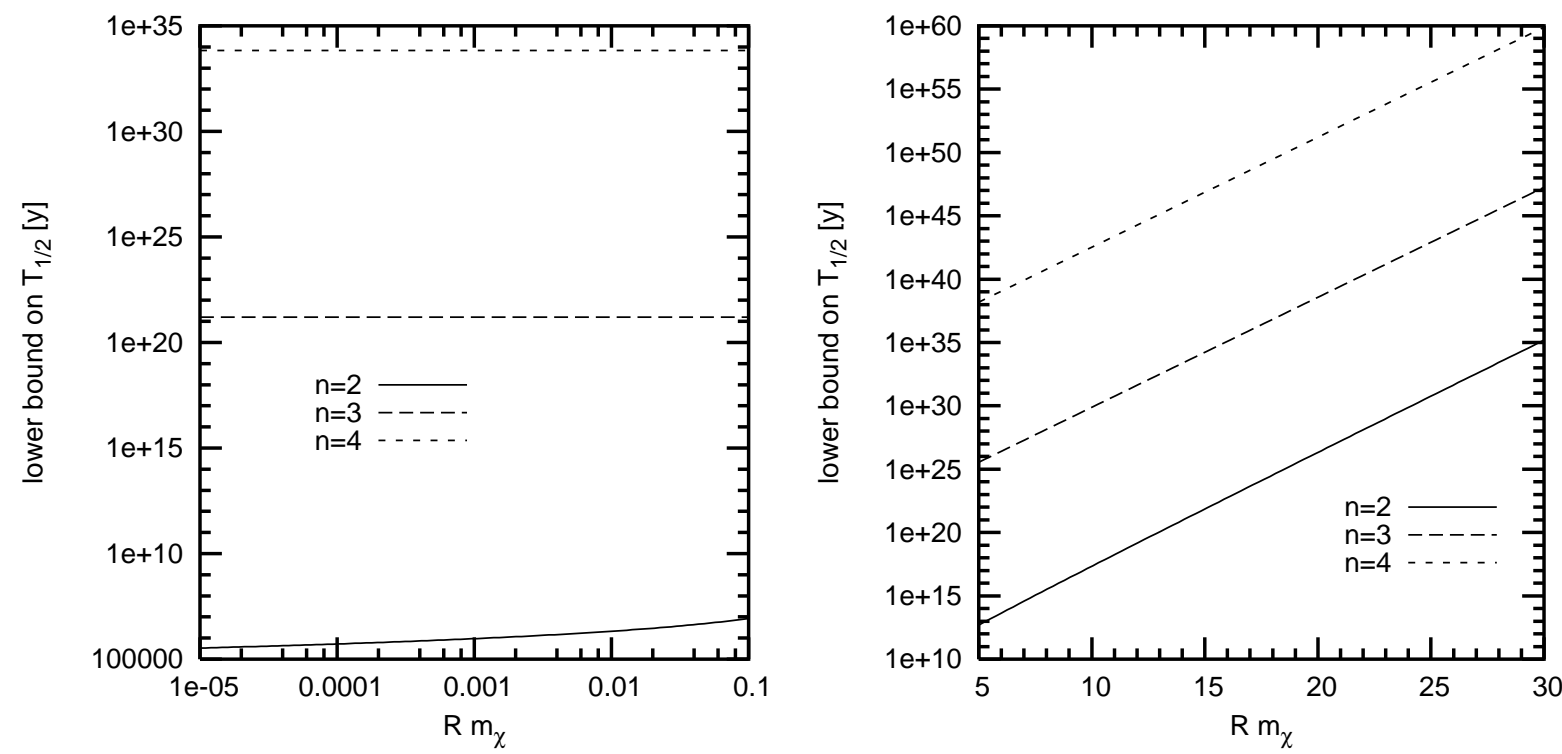

FIG. 2: Lower bounds on the half-life of neutrinoless double beta decay in the case of light (left panel) and heavy (right panel) messenger particle for the planned DCBA $\left({ }^{150} \mathrm{Nd}\right)$ experiment.

periments. In fact the values $R m_{\chi}$ around 3 correspond to the sensitivities of currently performed experiments, like the Germanium neutrinoless double beta decay in Gran Sasso. We repeat here, that for such small value, the validity of the results may be questioned. For light $\chi$ particle the calculations give $T_{1 / 2}>3.9 \times 10^{-2} \xi^{\prime}$, so there is no $m_{\chi}$ dependence. Since the experimental factor is of the order of $10^{24}$, we end up with $T_{1 / 2}>10^{22-23} \mathrm{y}$.

If the number of extra dimensions is four, a simple calculation gives $T_{1 / 2}>10^{38} \mathrm{y}$ for heavy messenger. This value is beyond the abilities of any currently planned ex- periment. It may, however, serve as clue if all of them report negative results. For a light messenger, we face a similar situation as previously, i.e. the $m_{\chi}$ dependence drops out. For four extra dimensions we finish with $T_{1 / 2}>1.69 \times 10^{11} \xi^{\prime}$, therefore obtaining $T_{1 / 2}>10^{35} \mathrm{y}$.

The discussion may be extended for more extra dimensions, if one is interested in the half-life of $0 \nu 2 \beta$ being longer than $10^{38}$ years. 

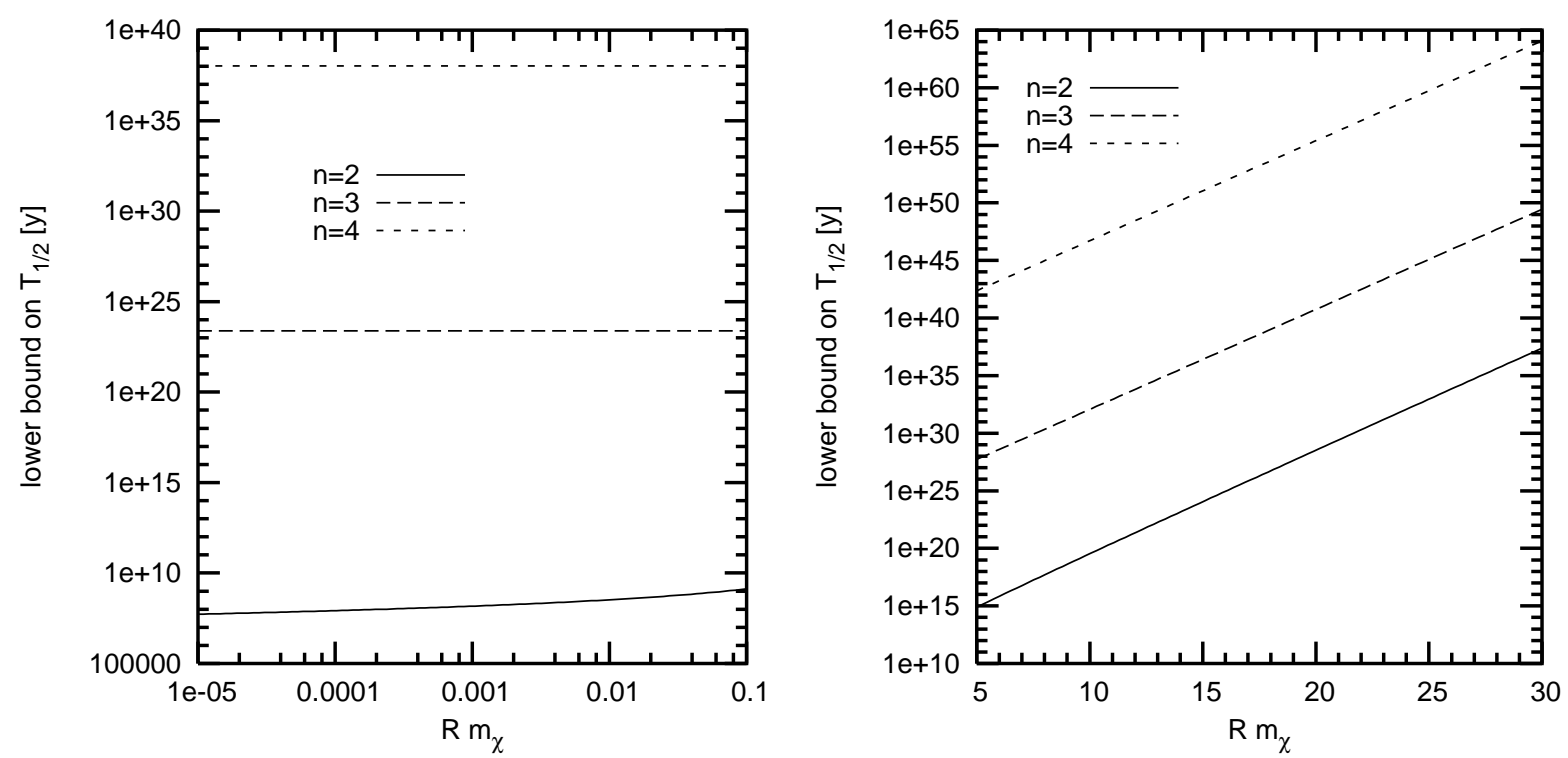

FIG. 3: Lower bounds on the half-life of neutrinoless double beta decay in the case of light (left panel) and heavy (right panel) messenger particle for the planned DAMA experiment.

TABLE II: Lower bounds on $T_{1 / 2}$ in years for two additional dimensions $\left(R<1.5 \times 10^{-7} \mathrm{~mm}\right)$.

\begin{tabular}{lccccccc}
\hline \hline$R m_{\chi}=$ & 0.001 & 0.01 & 0.1 & 10 & 15 & 20 \\
\hline DAMA & $1.6 \times 10^{8}$ & $3.6 \times 10^{8}$ & $1.4 \times 10^{9}$ & $3.7 \times 10^{19}$ & $1.2 \times 10^{24}$ & $3.6 \times 10^{28}$ & $1.0 \times 10^{33}$ \\
MAJORANA & $1.5 \times 10^{8}$ & $3.3 \times 10^{8}$ & $1.3 \times 10^{9}$ & $3.4 \times 10^{19}$ & $1.1 \times 10^{24}$ & $3.3 \times 10^{28}$ & $9.2 \times 10^{32}$ \\
EXO (10t) & $1.4 \times 10^{8}$ & $3.2 \times 10^{8}$ & $1.3 \times 10^{9}$ & $3.3 \times 10^{19}$ & $1.1 \times 10^{24}$ & $3.2 \times 10^{28}$ & $9.0 \times 10^{32}$ \\
GEM & $1.3 \times 10^{8}$ & $3.1 \times 10^{8}$ & $1.2 \times 10^{9}$ & $3.2 \times 10^{19}$ & $1.0 \times 10^{24}$ & $3.1 \times 10^{28}$ & $8.6 \times 10^{32}$ \\
GENIUS & $1.3 \times 10^{8}$ & $3.0 \times 10^{8}$ & $1.2 \times 10^{9}$ & $3.1 \times 10^{19}$ & $1.0 \times 10^{24}$ & $3.0 \times 10^{28}$ & $8.3 \times 10^{32}$ \\
CANDLES & $9.7 \times 10^{7}$ & $2.2 \times 10^{8}$ & $8.7 \times 10^{8}$ & $2.3 \times 10^{19}$ & $7.5 \times 10^{23}$ & $2.2 \times 10^{28}$ & $6.2 \times 10^{32}$ \\
MOON & $8.2 \times 10^{7}$ & $1.9 \times 10^{8}$ & $7.4 \times 10^{8}$ & $2.0 \times 10^{19}$ & $6.3 \times 10^{23}$ & $1.9 \times 10^{28}$ & $5.3 \times 10^{32}$ \\
XMASS & $7.2 \times 10^{7}$ & $1.7 \times 10^{8}$ & $6.5 \times 10^{8}$ & $1.7 \times 10^{19}$ & $5.6 \times 10^{23}$ & $1.7 \times 10^{28}$ & $4.7 \times 10^{32}$ \\
CUORE & $4.7 \times 10^{7}$ & $1.1 \times 10^{8}$ & $4.3 \times 10^{8}$ & $1.1 \times 10^{19}$ & $3.7 \times 10^{23}$ & $1.1 \times 10^{28}$ & $3.1 \times 10^{32}$ \\
COBRA & $2.4 \times 10^{7}$ & $5.5 \times 10^{7}$ & $2.1 \times 10^{8}$ & $5.7 \times 10^{18}$ & $1.8 \times 10^{23}$ & $5.5 \times 10^{27}$ & $1.5 \times 10^{32}$ \\
DCBA $\left({ }^{100} \mathrm{Mo}\right)$ & $2.3 \times 10^{7}$ & $5.4 \times 10^{7}$ & $2.1 \times 10^{8}$ & $5.6 \times 10^{18}$ & $1.8 \times 10^{23}$ & $5.4 \times 10^{27}$ & $1.5 \times 10^{32}$ \\
DCBA $\left({ }^{82} \mathrm{Se}\right)$ & $1.1 \times 10^{7}$ & $2.6 \times 10^{7}$ & $1.0 \times 10^{8}$ & $2.7 \times 10^{18}$ & $8.8 \times 10^{22}$ & $2.6 \times 10^{27}$ & $7.4 \times 10^{31}$ \\
CAMEO & $9.5 \times 10^{6}$ & $2.2 \times 10^{7}$ & $8.5 \times 10^{7}$ & $2.3 \times 10^{18}$ & $7.3 \times 10^{22}$ & $2.2 \times 10^{27}$ & $6.1 \times 10^{31}$ \\
DCBA $\left({ }^{150} \mathrm{Nd}\right)$ & $9.5 \times 10^{5}$ & $2.2 \times 10^{6}$ & $8.5 \times 10^{6}$ & $2.3 \times 10^{17}$ & $7.3 \times 10^{21}$ & $2.2 \times 10^{26}$ & $6.1 \times 10^{30}$ \\
\hline \hline
\end{tabular}

\section{SUMMARY}

Theories which deal with extra dimensions face one basic problem, namely the obvious difficulty of verification. There are two main types of experiments performed nowadays. One are the tabletop gravity experiments, which test the Newton $1 / r^{2}$ law on very small distances. The best accuracy of present setups is $0.1 \mathrm{~mm} \mathrm{38}$ ] which is not sufficient. The other are based on astronomical observations, mainly concerning supernovas and black holes [30 36]. These, however, are very difficult and highly model-dependent. The work presented first in Refs. 16 and [17], and continued here, shows that it is possible to use the results for exotic nuclear processes also to constrain the theories with large extra dimensions. Needless to say, we have much better control over these experiments, than those aforementioned.

To sum up the results of the present paper, one can say that within the model used, the current $0 \nu 2 \beta$ experiments have reached the sensitivity to explore the possibility of two, and maybe three additional spatial dimensions. The future experiments should be able to rule out the possibility of three extra dimensions, provided they finish with negative results. The sensitivity needed to reach the $n=4$ threshold should be at least $\sim 10^{35} \mathrm{y}$, which is 7 to 9 orders of magnitude better than the currently planned experiments can achieve. 
TABLE III: Lower bounds on $T_{1 / 2}$ in years for three additional dimensions $\left(R<2.6 \times 10^{-9} \mathrm{~mm}\right)$. The case of light $m_{\chi}$ is discussed in the text.

\begin{tabular}{lccc}
\hline \hline$R m_{\chi}=$ & 3 & 5 & 10 \\
\hline DAMA & $1.1 \times 10^{26}$ & $6.0 \times 10^{27}$ & $1.3 \times 10^{32}$ \\
MAJORANA & $1.0 \times 10^{26}$ & $5.5 \times 10^{27}$ & $1.2 \times 10^{32}$ \\
EXO $(10 \mathrm{t})$ & $1.0 \times 10^{26}$ & $5.4 \times 10^{27}$ & $1.2 \times 10^{32}$ \\
GEM & $9.5 \times 10^{25}$ & $5.2 \times 10^{27}$ & $1.1 \times 10^{32}$ \\
GENIUS & $9.1 \times 10^{25}$ & $5.0 \times 10^{27}$ & $1.1 \times 10^{32}$ \\
CANDLES & $6.8 \times 10^{25}$ & $3.7 \times 10^{27}$ & $8.1 \times 10^{31}$ \\
MOON & $5.8 \times 10^{25}$ & $3.2 \times 10^{27}$ & $6.9 \times 10^{31}$ \\
XMASS & $5.1 \times 10^{25}$ & $2.8 \times 10^{27}$ & $6.0 \times 10^{31}$ \\
CUORE & $3.4 \times 10^{25}$ & $1.8 \times 10^{27}$ & $4.0 \times 10^{31}$ \\
COBRA & $1.7 \times 10^{25}$ & $9.2 \times 10^{26}$ & $2.0 \times 10^{31}$ \\
DCBA $\left({ }^{100} \mathrm{Mo}\right)$ & $1.6 \times 10^{25}$ & $9.0 \times 10^{26}$ & $2.0 \times 10^{31}$ \\
DCBA $\left({ }^{82} \mathrm{Se}\right)$ & $8.1 \times 10^{24}$ & $4.4 \times 10^{26}$ & $9.6 \times 10^{30}$ \\
CAMEO & $6.7 \times 10^{24}$ & $3.6 \times 10^{26}$ & $8.0 \times 10^{30}$ \\
DCBA $\left({ }^{150} \mathrm{Nd}\right)$ & $6.7 \times 10^{23}$ & $3.6 \times 10^{25}$ & $8.0 \times 10^{29}$ \\
\hline \hline
\end{tabular}

\section{Acknowledgments}

This work has been supported by the Polish State Committee for Scientific Research under grants no. 2P03B 07125 and 1P03B 09827 and the Inter- national Graduiertenkolleg GRK683 by the DFG (Germany). Two of us (MG, WAK) would like to thank Prof. A. Faessler for his warm hospitality in Tübingen during the Summer 2004.
[1] Q. R. Ahmad et al. (SNO Collaboration), Phys. Rev. Lett. 89, 011301 (2002).

[2] Q. R. Ahmad et al. (SNO Collaboration), Phys. Rev. Lett. 89, 011302 (2002).

[3] S. N. Ahmed et al. (SNO Collaboration), Phys. Rev. Lett. 92, 181301 (2004).

[4] S. H. Ahn et al. (K2K-KEK Experiment), Phys. Rev. Lett. 90, 041801 (2003).

[5] Y. Ashie et al. (Super-Kamiokande Collaboration), Phys. Rev. Lett. 93, 101801 (2004).

[6] T. Kaluza, Preuss. Akad. Wiss., Phys.-Math. Kl., Berlin Math. Phys. Bd. K1, 966 (1921).

[7] O. Klein, Z. Phys. 37, 895 (1926).

[8] L. Randall and R. Sundrum, Phys. Rev. Lett. 83, 3370 (1999).

[9] L. Randall and R. Sundrum, Phys. Rev. Lett. 83, 4690 (1999).

[10] N. Arkani-Hamed, S. Dimopoulos, and G. Dvali, Phys. Lett. B 516, 70 (1998).

[11] N. Arkani-Hamed, S. Dimopoulos, and G. Dvali, Phys. Rev. D 59, 086004 (1999).

[12] I. Antoniadis, N. Arkani-Hamed, S. Dimopoulos, and G. Dvali, Phys. Lett. B 436, 257 (1998).

[13] N. Arkani-Hamed and S. Dimopoulos, Phys. Rev. D 65, 052003 (2002).

[14] N. Arkani-Hamed, S. Dimopoulos, G. Dvali, and J. March-Russell, Phys. Rev. D 65, 024032 (2002).

[15] M. Doi, T. Kotani, and E. Takasugi, Prog. of Theor. Phys. Suppl. 83, 1 (1985).
[16] M. Góźdź and W. A. Kamiński, Phys. Rev. D 68, 057901 (2003).

[17] M. Góźdź and W. A. Kamiński, Int. J. Mod. Phys. E13, 367 (2004).

[18] H. V. Klapdor-Kleingrothaus, A. Dietz, H. Harney, and I. Krivosheina, Mod. Phys. Lett. A 16, 2409 (2001).

[19] C. E. Aalseth et al., Mod. Phys. Lett. A 17, 1475 (2002).

[20] A. M. Bakalyarov et al. (2003), hep-ex/0309016.

[21] S. R. Elliott and P. Vogel, Annu. Rev. Nucl. Part. Sci. 52, 115 (2002).

[22] N. Ishihara, talk given at the APPI conference, Japan (2004).

[23] G. Gratta, talk given at the Lepton-Photon 03 conference, Fermilab, USA (2003).

[24] R. Bernabei et al. (DAMA Collaboration), Phys. Lett. B 546, 23 (2003).

[25] M. Danilov et al. (EXO Collaboration), Phys. Lett. B 480, 12 (2000).

[26] M. Apollonio et al., Phys. Lett. B 420, 397 (1998).

[27] M. Apollonio et al., Eur. Phys. J. C 27, 331 (2003).

[28] H. Päs and T. J. Weiler, talk given at the 3rd Workshop on Neutrino Oscillations and their Origin (NOON 2001), Kashiwa, Japan, hep-ph/0205191 (2001).

[29] S. M. Bilenky, A. Faessler, and F. Simkovic, Phys. Rev. D 70, 033003 (2004).

[30] S. Hannestad and G. Raffelt, Phys. Rev. Lett. 87, 051301 (2001).

[31] S. Hannestad and G. Raffelt, Phys. Rev. Lett. 88, 071301 (2002). 
[32] S. Hannestad and G. Raffelt, Phys. Rev. D 67, 125008 (2003).

[33] C. Hanhart et al., Nucl. Phys. B 595, 335 (2001).

[34] C. Hanhart et al., Phys. Lett. B 509, 1 (2001).

[35] L. J. Hall and D. R. Smith, Phys. Rev. D 60, 085008 (1999).
[36] S. Hannestad, Phys. Rev. D 64, 023515 (2001).

[37] H. V. Klapdor-Kleingrothaus, I. Krivosheina, A. Dietz, and O. Chkvorets, Phys. Lett. B 586, 198 (2004).

[38] J. C. Long et al., Nature 421, 922 (2003). 